\title{
A GPU-accelerated Performance Optimized RAP-MUSIC Algorithm for Real-Time Source Localization
}

C. Dinh, Institute of Biomedical Engineering and Informatics, Ilmenau University of Technology, Ilmenau, Germany, christoph.dinh@tu-ilmenau.de

J. Rühle, Department of Mathematics and Computer Sciences, Friedrich Schiller University Jena, Jena, Germany, johannes.ruehle@tu-ilmenau.de

S. Bollmann, Zentrum für MR-Forschung, Kinderspital Zürich, Zurich, Switzerland, steffen.bollmann@kispi.uzh.ch

J. Haueisen, Institute of Biomedical Engineering and Informatics, Ilmenau University of Technology, Germany, jens.haueisen@tu-ilmenau.de

D. Güllmar, Department of Radiology, Jena University Hospital, Jena, Germany, daniel.guellmar@med.uni-jena.de

\section{Introduction}

Up to now source localizations using scanning approaches, such as Recursively Applied and Projected Multiple Signal Classification (RAP-MUSIC), were hardly able to deliver real-time results due to their computational effort.

\section{Methods}

To realize RAP-MUSIC real-time source localization we reduce in a first step the computational costs by modifying and pre-calculating components of the subspace correlation. In a second step we apply to the accelerated algorithm a modified Powell's Conjugate Gradient Method, which highly optimizes the search process. Since the subspace correlations of the RAP-MUSIC algorithm are independent, they are predestined to be computed on a many-core processor such as a graphics processing unit (GPU) in parallel. For the parallelization, we utilize the GPU and NVIDIAs Compute Unified Device Architecture (CUDA). The robustness of the localization accuracy is proved by the help of simulations, where we analyze the influence of the SNR and different regularization parameters on the localization accuracy and calculation time.

\section{Results}

By modifying and pre-calculating components of the subspace correlation the computational costs are reduced by about 50 percent. These modifications together with Powell's Conjugate Gradient Method, allow the application for real-time source localizations. The algorithm provides the ability to obtain up to ten localizations per second with just one GPU (Tesla C2050) on a typically sized lead field matrix (10000 dipoles). The localization accuracy is depending on the SNR slightly lower compared to the original RAP-MUSIC, e.g. an average localization error of $12 \mathrm{~mm}$ compared to $9 \mathrm{~mm}$ at a SNR of $-5 \mathrm{~dB}$.

\section{Conclusion}

The results show that the presented RAP-MUSIC algorithm has fast and robust source localization accuracy and calculation times. Therefore it is feasible to localize neural sources in real-time with an ordinary workstation and a high-end GPU using RAP-MUSIC. 